\title{
Estimating distance, pressure, and dust opacity using submillimeter observations of self-gravitating filaments (Corrigendum)
}

\author{
J. Fischera and P. G. Martin \\ Canadian Institute for Theoretical Astrophysics, University of Toronto, 60 St. George Street, ON M5S3H8, Canada \\ e-mail: fischera@cita.utoronto.ca
}

A\&A 547, A86 (2012), DOI: 10.1051/0004-6361/201219728

Key words. methods: observational - techniques: photometric - ISM: clouds - dust, extinction - ISM: structure - errata, addenda

The formula for the flux density per unit length (Eq. (8) in the paper of Fischera \& Martin 2012) contained the product $\delta \eta_{v}$. Since the notation $\eta_{v}$ already incorporates $\delta$ implicitly, the correct form is

$F_{v}^{l}=\int \mathrm{d} \vartheta I_{v}^{\mathrm{fil}}(\vartheta)=\frac{M / l}{D \cos i} \eta_{v}=\frac{\eta_{v}}{D \cos i} f_{\mathrm{cyl}} \frac{2 K}{G}$.
This is a notational error, not used in our calculations, and so it does not affect any of the results.

\section{References}

(8) Fischera, J., \& Martin, P. G. 2012, A\&A, 547, A86 